OPEN ACCESS

Edited by:

J. Stephen Dumler, Uniformed Services University of the Health Sciences, United States

Reviewed by: Christian Keller,

Philipps-Universität Marburg, Germany Werner Solbach, Universität zu Lübeck, Germany

*Correspondence: Lynn Soong lysoong@utmb.edu

Specialty section: This article was submitted to Microbial Immunology, a section of the journa Frontiers in Microbiology

Received: 21 May 2019 Accepted: 22 August 2019 Published: 06 September 2019

Citation:

Trent B, Fisher J and Soong L (2019) Scrub Typhus Pathogenesis: Innate Immune Response and Lung Injury

During Orientia tsutsugamushi Infection.

Front. Microbiol. 10:2065 doi: 10.3389/fmicb.2019.02065

\section{Scrub Typhus Pathogenesis: Innate Immune Response and Lung Injury During Orientia tsutsugamushi Infection}

\author{
Brandon Trent ${ }^{1}$, James Fisher ${ }^{2}$ and Lynn Soong ${ }^{1,2 *}$ \\ ${ }^{1}$ Department of Pathology, University of Texas Medical Branch, Galveston, TX, United States, ${ }^{2}$ Department of Microbiology \\ and Immunology, University of Texas Medical Branch, Galveston, TX, United States
}

Scrub typhus is an understudied, potentially lethal disease caused by infection with Orientia tsutsugamushi. Despite causing an estimated 1 million cases per year and an increasing global presence, mechanisms of scrub typhus pathogenesis remain unclear. One of the most life-threatening conditions that can arise in scrub typhus patients is acute respiratory distress syndrome (ARDS). The development of ARDS is a complex process; some of its pathological hallmarks, including prolonged recruitment of inflammatory immune cells to the lung and vasculature damage, have been observed in humans and/or animal models of $O$. tsutsugamushi infection. Although different cell types and mechanisms may contribute to ARDS development during $O$. tsutsugamushi infection, this review highlights our current evidence of pulmonary endothelial activation and damage, the potential roles of neutrophils and macrophages in the lung, and the knowledge gaps in this field. Continued investigation of the lung microenvironment and cellular interactions will help elucidate disease pathogenesis and possible treatment during scrub typhus.

Keywords: Orientia tsutsugamushi, scrub typhus, acute respiratory distress syndrome, endothelial cell, neutrophil, macrophage

\section{INTRODUCTION}

The etiological agent of scrub typhus is Orientia tsutsugamushi, an obligate intracellular bacterium with unique biological features. Unlike other gram negative bacteria, O. tsutsugamushi lacks lipopolysaccharides and expresses low levels of unclassical peptidoglycans (Salje, 2017). It has a unique genome, with about $42 \%$ of its genetic content consisting of repeat DNA sequences and mobile genetic elements, making genetic manipulation challenging or unsuccessful (Salje, 2017). Despite approximately 1 million new cases every year, scrub typhus is a neglected tropical disease (Paris et al., 2012). The majority of cases occur in the "tsutsugamushi triangle," a region encompassing much of northern and eastern Asia, islands of the western Pacific Ocean, and a portion of northern Australia (Kelly et al., 2009). However, recent reports have identified cases of $O$. tsutsugamushi infection in areas previously thought free from scrub typhus, such as South America (Weitzel et al., 2016; Xu et al., 2017). Human infection is initiated via bite of an infected larval Leptotrombidium mite (also known as a chigger). After an incubation period (6-21 days), eschar, fever, headache, and malaise may develop 
(Kundavaram et al., 2013; Peter et al., 2015). Unless treated quickly, the bacteria can spread systemically, causing interstitial pneumonia, myocardial and hepatic lesions, meningoencephalitis, acute respiratory distress syndrome (ARDS), and multi-organ failure (Peter et al., 2015). Indirect fluorescent antibody (IFA) testing is the diagnostic gold standard and becoming more widely available in high incidence countries; however, IFA must be performed with care due to high-incidence areas having pre-existing immunity to scrub typhus (Blacksell et al., 2007). No vaccine exists for O. tsutsugamushi, and poor or limited cross-protection between heterologous Orientia strains is of particular concern (Valbuena and Walker, 2012).

Lung infection is common during scrub typhus, and patients typically develop mild interstitial pneumonitis during selfresolving or promptly treated scrub typhus (Song et al., 2004). However, in severe cases, pulmonary pathology includes lung hemorrhage, pulmonary edema, vasculature damage, and diffuse cellular infiltration resulting in ARDS (Jeong et al., 2007; Hsu and Chen, 2008). The development of ARDS is a complex process initiated by direct or indirect lung injury (Longo and Harrison, 2012). Damage to lung endothelial cells (ECs) can lead to accumulation of protein-rich edema in the interstitial and alveolar space, producing characteristic signs and symptoms: progressive dyspnea, hypoxemia, and alveolar infiltrates visible on chest X-ray (Siegel, 2019). Once alternative etiologies are excluded, a patient must meet the following criteria to be diagnosed with ARDS, according to the Berlin definition: respiratory symptoms beginning or worsening within 1 week of an established insult, the presence of bilateral opacities on chest $\mathrm{x}$-ray or CT scan, and moderate-to-severe reduction in oxygenation capacity as measured by the $\mathrm{PaO}_{2} / \mathrm{FiO}_{2}$ fraction (Force et al., 2012). Damage to the vasculature promotes the production of proinflammatory cytokines (i.e., CXCL8 and $\mathrm{TNF} \alpha$ ) leading to recruitment of leukocytes, especially neutrophils, which secrete their own chemokines and effector proteins, inducing a proinflammatory microenvironment. Without resolution of inflammation and/or prompt treatment, atelectasis and death may occur (Longo and Harrison, 2012).

In scrub typhus, about 6-44\% of cases develop ARDS (Wang et al., 2007; Varghese et al., 2013, 2014; Jayasimha et al., 2017). This wide incidence range may be due to different regional strains of $O$. tsutsugamushi, differences in disease detection time, population genetics (Xu et al., 2017), or employment of different diagnostic criteria for ARDS. Both Wang et al. and Jayasimha et al. utilized internationally accepted diagnostic definitions and reported ARDS incidence rates of approximately 11 and 6\%, respectively, in scrub typhus patients (Wang et al., 2007; Jayasimha et al., 2017). Publications not stating diagnostic criteria tended to have higher observed incidence rates (Varghese et al., 2013, 2014). Despite these differences, certain risk factors appear to be important for ARDS development during scrub typhus: septic shock, hypoalbuminemia, high circulating white blood cell counts, delayed antibiotic treatment, and advanced age (Tsay and Chang, 2002; Jayasimha et al., 2017). Scrub typhus patients that developed ARDS presented with many clinical features, including pleural effusion, peribronchial thickening, hyaline membrane formation, and occasionally, pulmonary edema (Hsu and Chen, 2008; Abhilash et al., 2016; Sahoo et al., 2016).

Considering the life-threatening complications of ARDS, establishing animal models of this syndrome has garnered significant effort. Murine models of ARDS demonstrate histologic evidence of tissue injury, changes in the alveolar capillary barrier, inflammatory infiltration, and physiologic dysfunction (Aeffner et al., 2015). These models have led to significant advances in understanding the pathophysiology of ARDS; but, there are inherent limiting factors in comparing mouse models of ARDS to humans (Aeffner et al., 2015).

Few studies have investigated scrub typhus-related ARDS, leaving fundamental questions unanswered. We and other groups have recently established a number of murine models of $O$. tsutsugamushi infection mimicking severities of scrub typhus observed in humans (Keller et al., 2014; Shelite et al., 2014; Mendell et al., 2017). Both self-healing and lethal infection models have been established, but pathologies firmly delineating disease outcomes have yet to be uncovered. Murine models, coupled with known human infection data, are now being used to answer questions regarding tissue pathology and immune response during infection. Here, we summarize recent studies regarding pulmonary involvement of several cell types during $O$. tsutsugamushi infection that play key roles in lung injury, focusing on ECs, neutrophils, and macrophages (MФ). How these three types of immune cells interact with and respond to O. tsutsugamushi infection and their contribution to disease progression is critical for the development of effective management strategies.

\section{ENDOTHELIAL CELLS}

ECs are one of the primary cellular targets of O. tsutsugamushi during systemic infection. Using autopsy tissues from U.S. soldiers who died of scrub typhus, Moron and colleagues reported $O$. tsutsugamushi in ECs of infected tissues, including the lung (Moron et al., 2001). While in vitro evidence exists for O. tsutsugamushi-induced activation of human ECs and cell death (Kim et al., 1999; Cho et al., 2001, 2010), there are no detailed reports for the mechanisms determining ARDS development in scrub typhus. It is well documented from other severe diseases that EC activation and damage are hallmarks of ARDS development (Matthay and Zemans, 2011; Gonzales et al., 2015). EC activation can be triggered directly via pathogen replication and the recognition of pathogen associated molecular patterns, or indirectly via recognition of damage-associated molecular patterns (DAMP) and inflammatory cytokines. EC activation promotes leukocyte adhesion/transmigration, antigen presentation, and cytokine production (Orfanos et al., 2004; Mai et al., 2013). However, uncontrolled EC activation results in excessive influx of neutrophils and $M \Phi$ s/monocytes to the lung interstitium. This influx leads to increased vascular permeability resulting in edema, tissue hypoxemia, and ARDS (Silliman et al., 2007; Gill et al., 2015; Moussa et al., 2015).

For animal models of scrub typhus, few studies document EC activation and injury. Since 2014, our group has documented pulmonary EC cellular tropism in C57BL/6 mice infected with 
O. tsutsugamushi Karp strain via the intravenous (i.v.) (Shelite et al., 2014) or intradermal (i.d.) inoculation route (Soong et al., 2016; Mendell et al., 2017). Regardless of route of inoculation, the lungs carry the higher bacterial loads than the liver, spleen, kidney, and brain (Shelite et al., 2014; Soong et al., 2016; Mendell et al., 2017). Likewise, Keller and colleagues used a footpad inoculation of $O$. tsutsugamushi Karp strain in $\mathrm{BALB} / \mathrm{c}$ mice and confirmed that the lungs carry the highest bacterial loads. However, bacteria were interpreted to be localized within pulmonary MФs, rather than in pulmonary ECs (Keller et al., 2014). At present, it is unclear whether O. tsutsugamushi preferentially replicates within ECs or phagocytes at different stages in mouse models; future development of reporter bacteria would be valuable in such research.

Sublethal infection in mice via i.d. inoculation of $O$. tsutsugamushi Karp, Gilliam, or Woods strains resulted in increased circulation of EC activation markers such as soluble VCAM-1 and soluble ICAM-1 (Sunyakumthorn et al., 2013). The sICAM-1 levels were also significantly increased following i.d. inoculation of rhesus macaques (Sunyakumthorn et al., 2018). Clinical data are consistent with animal models, as sVCAM-1 and sALCAM (soluble activated leukocyte cell adhesion molecule) are increased in scrub typhus patients compared to healthy controls and are correlated with organ dysfunction (Otterdal et al., 2014). However, serum concentration measurements provide little in the context of pulmonary-specific endothelial activation/dysfunction. Use of immunofluorescent staining on infected murine lung tissue would allow visualization of increased EC markers in vivo (Kim et al., 2016). More importantly, O. tsutsugamushi-infected, lung-derived cells would be of great value for flow cytometric analyses of alterations in EC-specific markers and EC viability or gene profile analyses, as in other infection models (Singer et al., 2016; Lai et al., 2018; Lukowski et al., 2019).

Given the evident EC activation, it is speculated that $O$. tsutsugamushi infection can trigger the expression/release of DAMP molecules, and our studies with infected human ECs and mouse tissues suggest that a pathogenic role of IL-33 (Shelite et al., 2016), a nucleus-located alarmin, belongs to the IL-1 family. It is known from other model systems that IL-33 released from damaged ECs can be processed and activated extracellularly via cleavage by enzymes such as neutrophil elastase or cathepsin G (Lefrancais et al., 2012), acting as a DAMP molecules on nearby EC (Cayrol and Girard, 2009; Gautier et al., 2016). IL-33 ${ }^{-/-}$mice demonstrated significantly less weight loss during lethal infection with O. tsutsugamushi, and exogenous recombinant IL-33 treatment had exacerbated disease following sublethal infection (Shelite et al., 2016). Complementing these findings, neutralizing IL-33 antibody was shown to reduce inflammation and lung injury in the murine LPS-induced ARDS model (Lin et al., 2016). Currently, there is no evidence of IL-33 production in pulmonary ECs or alveolar epithelium in O. tsutsugamushi-infected mice. The utilization of IL-33 reporter mice (Heyen et al., 2016) will help examine cellular sources and kinetics of IL-33 production correlating to local pathology during O. tsutsugamushi infection and disease pathology.
For EC-related biomarkers in severe O. tsutsugamushi infection, angiopoietin 2 (Ang2) and angiopoietin 1 (Ang1) levels and their expression ratio are highly relevant (van Meurs et al., 2009; Parikh, 2017). While Ang1 is constitutively expressed by pericytes to promote EC quiescence and effective barrier function (van Meurs et al., 2009), Ang2 is released/produced by ECs during activation or damage. Both molecules compete to bind Tie2 in order to modulate EC function (van Meurs et al., 2009). There are reports that increased Ang2 levels correlate with ARDS development in malaria (Ghosh et al., 2016; Higgins et al., 2016), influenza (Ghosh et al., 2016), and sepsis (David et al., 2011; Stiehl et al., 2014; Ghosh et al., 2016). Our O. tsutsugamushiinfected mouse models have consistently shown an increased Ang2/Ang1 mRNA ratio in the lung, liver, and brain tissues at the onset of disease and prior to host death (Soong et al., 2014, 2017). Whether tissue and serum levels of Ang2/Ang1 correlate with human scrub typhus patients has yet to be determined.

Our current understanding of $O$. tsutsugamushi infectioninduced EC activation or injury is limited, however, by examining gene expression of primary human EC cultures and mouse lung tissue homogenates (Soong et al., 2014; Shelite et al., 2016). Studies to determine changes in pulmonary endothelium during infection would benefit from more honed approaches, such as the use of single-cell RNA sequencing to characterize pathways important to both EC activation and homeostasis.

\section{NEUTROPHILS}

Neutrophils are the most abundant leukocyte in circulation and are considered the "first responders" during tissue injury or infection (Summers et al., 2010). Neutrophil recruitment plays an important function in early control of human pathogens (Bou Ghanem et al., 2015; Witter et al., 2016), but prolonged neutrophil involvement can be detrimental to host health (Saffarzadeh et al., 2012; Bou Ghanem et al., 2015). Neutrophil effector functions include phagocytosis and killing of bacteria, release of antimicrobial granule contents, and formation of neutrophil extracellular traps (NETs). The latter two functions work to combat pathogens but can also damage host tissues (Grommes and Soehnlein, 2011). In other model systems, NET formation in the lung is known to promote ARDS development, partially via increased epithelial and endothelial cytotoxicity (Saffarzadeh et al., 2012). Mice treated with DNase I or deletion of the PADI4 gene have reduced lung injury in a murine model of bacterial pneumonia-induced ARDS, at least in part due to reduced NET formation (Lefrançais et al., 2018).

Few studies have addressed the role of neutrophils during scrub typhus. Early in vitro work documented the presence of O. tsutsugamushi in phagosomes within neutrophils, as well as in the extracellular space (Rikihisa and Ito, 1982). However, despite such findings nearly 40 years ago, it remains unclear if neutrophils are capable of killing the engulfed bacterium or if neutrophils provide another cellular niche for bacterial growth. It is known that neutrophils are detected at the site of the chigger bite in humans and that neutrophilia occurs during acute infection (Cho et al., 2012; Paris et al., 2012). Scrub typhus patients have increased 
CXCL8, a potent neutrophil chemoattractant, and its serum levels correlated with disease severity and mortality (Astrup et al., 2014). CXCL8 is increased in individuals with ARDS and is believed to contribute to ARDS pathology via neutrophil recruitment, inhibiting neutrophil apoptosis and activating lung endothelium (Chollet-Martin et al., 1993; Allen and Kurdowska, 2014). Importantly, Paris et al. showed that markers of neutrophil activation and possible NET formation were also significantly upregulated in the plasma of severe scrub typhus patients compared to less severe scrub typhus cases (Paris et al., 2015; Park et al., 2018).

Neutrophil activation and pulmonary recruitment during $O$. tsutsugamushi infection have been primarily studied in murine models. Immunohistochemical studies in both footpad and i.v. infection models revealed neutrophil infiltration of the pulmonary interstitium (Keller et al., 2014; Soong et al., 2014). Lung tissues from infected mice are strongly positive for myeloperoxidase (MPO; Soong et al., 2014), a peroxidase enzyme abundantly present in neutrophil primary granules that act as an antimicrobial. Since mice i.v. inoculated with $O$. tsutsugamushi have marked increase in CXCL1 and CXCL2 transcripts at the onset of disease (day 6) and prior to host death (Soong et al., 2014), the use of genetargeted knockout mice, as well as specific inhibitors or antibodies to block these chemokines or CXCR2, will help dissect protective versus pathogenic role of neutrophils in scrub typhus (Sônego et al., 2016). More research is needed to examine O. tsutsugamushi killing or growth within neutrophils; such information will elucidate bacterium-neutrophil interactions and their contribution to bacterial control versus dissemination. The contribution of specific neutrophil processes (e.g., degranulation and NET formation) to lung injury in scrub typhus should be investigated.

\section{MACROPHAGES}

Both resident alveolar MФs and recruited circulating monocytes are known to play important roles in the development and resolution of ARDS in other infections (Huang et al., 2018). For example, in vivo depletion models have demonstrated that $\mathrm{M} \Phi$ s can play either a pathogenic or a protective role during ARDS, depending on the method of lung injury induction and time of disease progression (Broug-Holub et al., 1997; Eyal et al., 2007; Narasaraju et al., 2011). MФs recruited to the lungs during ARDS have been shown to adopt an initial M1 profile, generating an acute inflammatory response and combating bacterial pathogens (Benoit et al., 2008; Aggarwal et al., 2014; D'Alessio et al., 2016). M1 polarization is believed to be partially responsible for initiation of ARDS via secretion of inflammatory cytokines and chemokines and production of reactive oxygen and nitrogen species (Aggarwal et al., 2014; Lu et al., 2018). Shifting the pulmonary $М \Phi$ population from M1 to an alternative M2 phenotype is understood to be important for resolution of lung inflammation and tissue healing (Aggarwal et al., 2014; Vergadi et al., 2014; D’Alessio et al., 2016).

During O. tsutsugamushi infection, МФs can serve not only as a cellular target of bacterial replication but also as an inflammatory response initiator (Cho et al., 2000; Moron et al., 2001). In vitro infection of MФs by O. tsutsugamushi can trigger inflammasome activation (Koo et al., 2012), initiate inflammatory signaling cascades (Yun et al., 2009), and generate chemokines for recruiting $\mathrm{T}$ cells, neutrophils, and monocytes (Cho et al., 2000). In humans (Paris et al., 2012) and rhesus macaque (Sunyakumthorn et al., 2013), infection of MФs is observed early in eschars and may propagate lymphogenous dissemination.

The recruitment and role of pulmonary MФs during systemic O. tsutsugamushi infection have been investigated using various murine models of infection. Self-resolving footpad inoculation generates a systemic infection with recruited MФs in the pleura and bronchial alveolar lymphatic tissue (Keller et al., 2014). Furthermore, O. tsutsugamushi was present in interstitial MФs, and iNOS (inducible nitric oxide) production was induced in the lung following infection (Keller et al., 2014). In congruence with animal findings, iNOS production has been observed in interstitial MФs from patients who succumbed to ARDS during severe scrub typhus (Hsu and Chen, 2008). Generation of iNOS to combat invading pathogens is a product of $M \Phi$ activation and is associated with M1 polarization. iNOS inhibition in mouse macrophages was associated with increased macrophage bacterial loads (Keller et al., 2014). The role of iNOS production in $\mathrm{M} \Phi$ control of O. tsutsugamushi is still somewhat uncertain, however, as others have reported increased bacterial loads within $M \Phi$ during prolonged infection that appears linked to iNOS production (Ogawa et al., 2017). Evidence of inflammatory M1 polarization is confirmed by transcriptome analysis of human monocytes and peripheral blood mononuclear cells from scrub typhus patients (Tantibhedhyangkul et al., 2011). While M1 polarization is known to contribute to lung injury and ARDS development (Aggarwal et al., 2014), the contribution of M1-polarized MФs to lung injury during scrub typhus is still unclear. Furthermore, the presence/role of M2 MФs during O. tsutsugamushi infection has not been explored. Future studies classifying $M \Phi$ subsets present in the lung and identifying their contribution to inflammation and tissue health during scrub typhus will be important to understand disease pathogenesis. Use of in vivo models with conditional knockout genes in MФs for iNOS production (McNeill et al., 2015) or specific M2-type genes, such as arginase 1 (Suwanpradid et al., 2017), may help us understand the role of $M \Phi$ polarization in bacterial dissemination and disease progression.

\section{CONCLUDING REMARKS}

Although considered a neglected pathogen, O. tsutsugamushi infection and disease burden in endemic areas have become better appreciated in recent years (Bonell et al., 2017). Lifethreatening complications like ARDS can quickly arise in scrub typhus patients not treated promptly. Therefore, it is important to understand mechanisms contributing to ARDS development during O. tsutsugamushi infection. This review highlights what is known regarding vascular activation and the innate immune response during scrub typhus (Figure 1). While adaptive immune cell subsets $\left(\mathrm{CD}^{+}\right.$and $\mathrm{CD}^{+} \mathrm{T}$ cells and Foxp $3^{+}$regulatory $\mathrm{T}$ cells) are not illustrated in Figure 1, their roles in O. tsutsugamushi infection need to be examined, given their important roles in 
ARDS development in other infection models (Adamzik et al., 2013; Risso et al., 2015; Li et al., 2016). Murine models of scrub typhus will help dissect key immune components in controlling O. tsutsugamushi infection and host factors and mechanisms underlying cellular injury and ARDS development in severe scrub typhus.

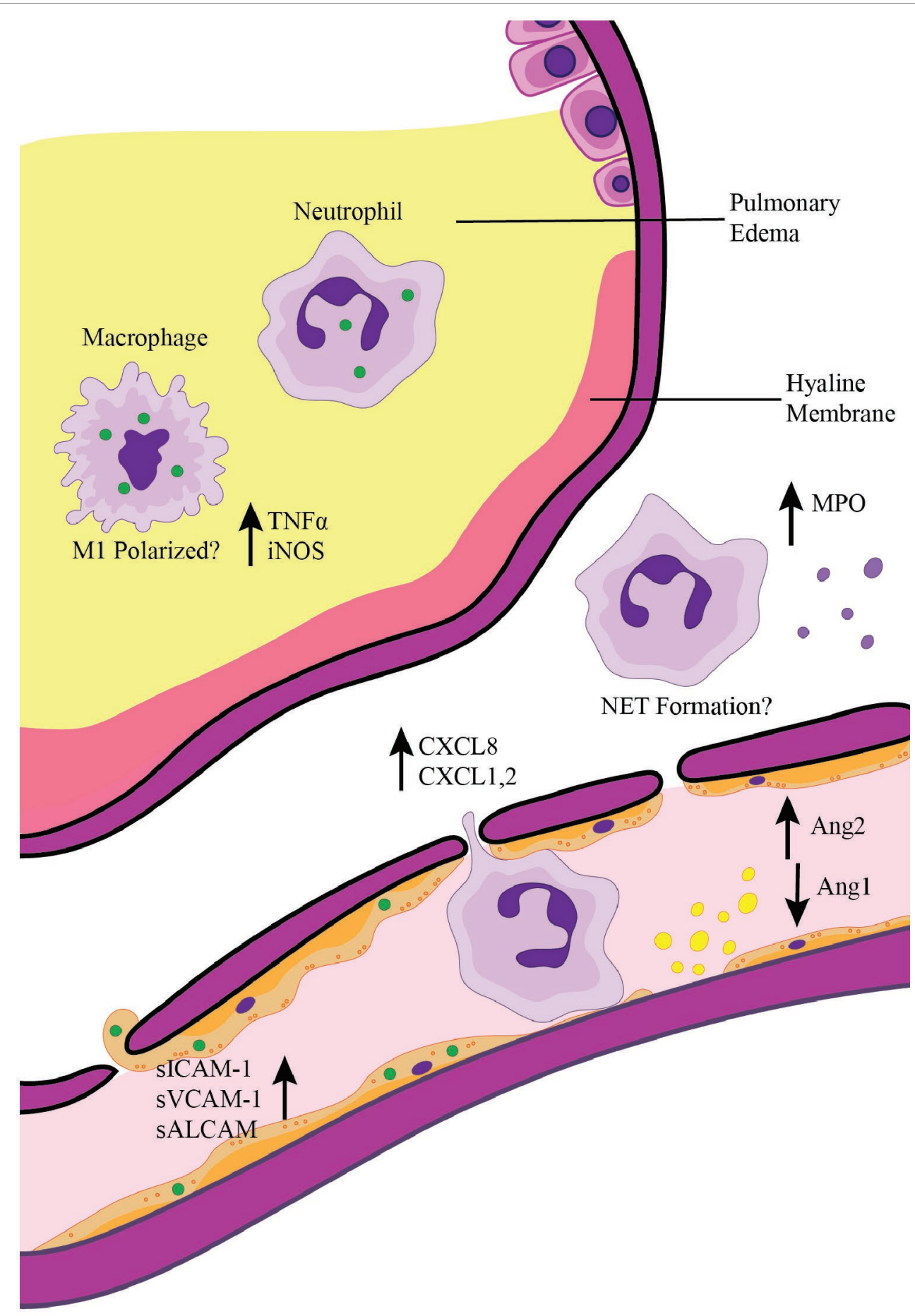

FIGURE 1 | Pulmonary inflammation and ARDS development during scrub typhus. Activation of pulmonary endothelial cells via direct infection with $O$. tsutsugamushi (green circles) or by interaction with inflammatory cytokines leads to the release of Ang2 and upregulation of adhesion molecules. Endothelial activation results in recruitment of neutrophils and macrophages, which can in turn secrete chemokines for further immune cell recruitment (i.e., CXCL1, CXCL2, and CXCL8). Infection of endothelial cells coupled with continued recruitment of inflammatory immune cells results in breakdown of the endothelial barrier, pleural effusion, and pulmonary edema seen in severe scrub typhus patients. Activation of recruited neutrophils and macrophages contributes to the inflammatory pulmonary environment. 


\section{AUTHOR CONTRIBUTIONS}

BT conceived and drafted the manuscript and conceived and organized the figure. JF and BT revised and edited the manuscript. LS revised and edited the manuscript and figure.

\section{FUNDING}

This work was partially supported by generous funding support was provided by the NIAID T32 Biodefense Training Program

\section{REFERENCES}

Abhilash, K., Mannam, P. R., Rajendran, K., John, R. A., and Ramasami, P. (2016). Chest radiographic manifestations of scrub typhus. J. Postgrad. Med. 62, 235-238. doi: 10.4103/0022-3859.184662

Adamzik, M., Broll, J., Steinmann, J., Westendorf, A. M., Rehfeld, I., Kreissig, C., et al. (2013). An increased alveolar CD $4+$ CD $25+$ Foxp $3+$ T-regulatory cell ratio in acute respiratory distress syndrome is associated with increased 30-day mortality. Intensive Care Med. 39, 1743-1751. doi: 10.1007/s00134-013-3036-3

Aeffner, F., Bolon, B., and Davis, I. C. (2015). Mouse models of acute respiratory distress syndrome: a review of analytical approaches, pathologic features, and common measurements. Toxicol. Pathol. 43, 1074-1092. doi: $10.1177 / 0192623315598399$

Aggarwal, N. R., King, L. S., and D’Alessio, F. R. (2014). Diverse macrophage populations mediate acute lung inflammation and resolution. Am. J. Physiol. Lung Cell. Mol. Physiol. 306, L709-L725. doi: 10.1152/ajplung.00341.2013

Allen, T. C., and Kurdowska, A. (2014). Interleukin 8 and acute lung injury. Arch. Pathol. Lab. Med. 138, 266-269. doi: 10.5858/arpa.2013-0182-RA

Astrup, E., Janardhanan, J., Otterdal, K., Ueland, T., Prakash, J. A., Lekva, T., et al. (2014). Cytokine network in scrub typhus: high levels of interleukin-8 are associated with disease severity and mortality. PLoS Negl. Trop. Dis. 8:e2648. doi: 10.1371/journal.pntd.0002648

Benoit, M., Desnues, B., and Mege, J. L. (2008). Macrophage polarization in bacterial infections. J. Immunol. 181, 3733-3739. doi: 10.4049/ jimmunol.181.6.3733

Blacksell, S. D., Bryant, N. J., Paris, D. H., Doust, J. A., Sakoda, Y., and Day, N. P. (2007). Scrub typhus serologic testing with the indirect immunofluorescence method as a diagnostic gold standard: a lack of consensus leads to a lot of confusion. Clin. Infect. Dis. 44, 391-401. doi: 10.1086/510585

Bonell, A., Lubell, Y., Newton, P. N., Crump, J. A., and Paris, D. H. (2017). Estimating the burden of scrub typhus: a systematic review. PLoS Negl. Trop. Dis. 11:e0005838. doi: 10.1371/journal.pntd.0005838

Bou Ghanem, E. N., Clark, S., Roggensack, S. E., McIver, S. R., Alcaide, P., Haydon, P. G., et al. (2015). Extracellular adenosine protects against Streptococcus pneumoniae lung infection by regulating pulmonary neutrophil recruitment. PLoS Pathog. 11:e1005126. doi: 10.1371/journal.ppat.1005126

Broug-Holub, E., Toews, G. B., van Iwaarden, J. F., Strieter, R. M., Kunkel, S. L., Paine, R., et al. (1997). Alveolar macrophages are required for protective pulmonary defenses in murine Klebsiella pneumonia: elimination of alveolar macrophages increases neutrophil recruitment but decreases bacterial clearance and survival. Infect. Immun. 65, 1139-1146.

Cayrol, C., and Girard, J. P. (2009). The IL-1-like cytokine IL-33 is inactivated after maturation by caspase-1. Proc. Natl. Acad. Sci. USA 106, 9021-9026. doi: 10.1073/pnas.0812690106

Cho, K. A., Jun, Y. H., Suh, J. W., Kang, J. S., Choi, H. J., and Woo, S. Y. (2010). Orientia tsutsugamushi induced endothelial cell activation via the NOD1-IL-32 pathway. Microb. Pathog. 49, 95-104. doi: 10.1016/j.micpath.2010.05.001

Cho, B. A., Ko, Y., Kim, Y. S., Kim, S., Choi, M. S., Kim, I. S., et al. (2012). Phenotypic characterization of peripheral $\mathrm{T}$ cells and their dynamics in scrub typhus patients. PLoS Negl. Trop. Dis. 6:e1789. doi: 10.1371/journal. pntd.0001789

Cho, N. H., Seong, S. Y., Choi, M. S., and Kim, I. S. (2001). Expression of chemokine genes in human dermal microvascular endothelial cell lines
(AI060549), 2018 AAI Careers in Immunology Fellowship (to BT and LS), and NIAID grants (AI132674 and AI126343 to LS).

\section{ACKNOWLEDGMENTS}

We would like to thank Dr. David Walker, Dr. Sanjeev Sanhi, and Dr. Yuejin Liang for assistance in editing and revising of this manuscript. We would also like to thank Camille Trent for her assistance in figure design.

infected with Orientia tsutsugamushi. Infect. Immun. 69, 1265-1272. doi: 10.1128/IAI.69.3.1265-1272.2001

Cho, N. H., Seong, S. Y., Huh, M. S., Han, T. H., Koh, Y. S., Choi, M. S. et al. (2000). Expression of chemokine genes in murine macrophages infected with Orientia tsutsugamushi. Infect. Immun. 68, 594-602. doi: 10.1128/ IAI.68.2.594-602.2000

Chollet-Martin, S., Montravers, P., Gibert, C., Elbim, C., Desmonts, J. M., Fagon, J. Y., et al. (1993). High levels of interleukin-8 in the blood and alveolar spaces of patients with pneumonia and adult respiratory distress syndrome. Infect. Immun. 61, 4553-4559.

D’Alessio, F. R., Craig, J. M., Singer, B. D., Files, D. C., Mock, J. R., Garibaldi, B. T. et al. (2016). Enhanced resolution of experimental ARDS through IL-4mediated lung macrophage reprogramming. Am. J. Physiol. Lung Cell. Mol. Physiol. 310, L733-L746. doi: 10.1152/ajplung.00419.2015

David, S., Park, J. K., Meurs, M., Zijlstra, J. G., Koenecke, C., Schrimpf, C. et al. (2011). Acute administration of recombinant angiopoietin-1 ameliorates multiple-organ dysfunction syndrome and improves survival in murine sepsis. Cytokine 55, 251-259. doi: 10.1016/j.cyto.2011.04.005

Eyal, F. G., Hamm, C. R., and Parker, J. C. (2007). Reduction in alveolar macrophages attenuates acute ventilator induced lung injury in rats. Intensive Care Med. 33, 1212-1218. doi: 10.1007/s00134-007-0651-x

Force, A. D. T., Ranieri, V. M., Rubenfeld, G. D., Thompson, B. T., Ferguson, N. D., Caldwell, E., et al. (2012). Acute respiratory distress syndrome: the Berlin definition. JAMA 307, 2526-2533. doi: 10.1001/jama.2012.5669

Gautier, V., Cayrol, C., Farache, D., Roga, S., Monsarrat, B., Burlet-Schiltz, O. et al. (2016). Extracellular IL-33 cytokine, but not endogenous nuclear IL-33, regulates protein expression in endothelial cells. Sci. Rep. 6:34255. doi: $10.1038 /$ srep34255

Ghosh, C. C., David, S., Zhang, R., Berghelli, A., Milam, K., Higgins, S. J., et al. (2016). Gene control of tyrosine kinase TIE2 and vascular manifestations of infections. Proc. Natl. Acad. Sci. USA 113, 2472-2477. doi: 10.1073/ pnas. 1519467113

Gill, S. E., Rohan, M., and Mehta, S. (2015). Role of pulmonary microvascular endothelial cell apoptosis in murine sepsis-induced lung injury in vivo. Respir. Res. 16:109. doi: 10.1186/s12931-015-0266-7

Gonzales, J. N., Lucas, R., and Verin, A. D. (2015). The acute respiratory distress syndrome: mechanisms and perspective therapeutic approaches. Austin J. Vasc. Med. 2.

Grommes, J., and Soehnlein, O. (2011). Contribution of neutrophils to acute lung injury. Mol. Med. 17, 293-307. doi: 10.2119/molmed.2010.00138

Heyen, L., Muller, U., Siegemund, S., Schulze, B., Protschka, M., Alber, G., et al. (2016). Lung epithelium is the major source of IL-33 and is regulated by IL-33-dependent and IL-33-independent mechanisms in pulmonary cryptococcosis. Pathog. Dis. 74, 1-11. doi: 10.1093/femspd/ftw086

Higgins, S. J., Purcell, L. A., Silver, K. L., Tran, V., Crowley, V., Hawkes, M. et al. (2016). Dysregulation of angiopoietin-1 plays a mechanistic role in the pathogenesis of cerebral malaria. Sci. Transl. Med. 8:358ra128. doi: $10.1126 /$ scitranslmed.aaf6812

Hsu, Y. H., and Chen, H. I. (2008). Pulmonary pathology in patients associated with scrub typhus. Pathology 40, 268-271. doi: 10.1080/00313020801911488

Huang, X., Xiu, H., Zhang, S., and Zhang, G. (2018). The role of macrophages in the pathogenesis of ALI/ARDS. Mediat. Inflamm. 2018:1264913. doi: $10.1155 / 2018 / 1264913$ 
Jayasimha, K., Varma, M., Kamath, A., Bairy, I., Singh, R., Mounika, S., et al. (2017). Risk factors of acute respiratory distress syndrome in scrub typhus. Int. J. Res. Med. Sci. 5, 3912-3915. doi: 10.18203/2320-6012.ijrms 20173722

Jeong, Y. J., Kim, S., Wook, Y. D., Lee, J. W., Kim, K. I., and Lee, S. H. (2007). Scrub typhus: clinical, pathologic, and imaging findings. Radiographics 27, 161-172. doi: $10.1148 / \mathrm{rg} .271065074$

Keller, C. A., Hauptmann, M., Kolbaum, J., Gharaibeh, M., Neumann, M., Glatzel, M., et al. (2014). Dissemination of Orientia tsutsugamushi and inflammatory responses in a murine model of scrub typhus. PLoS Negl. Trop. Dis. 8:e3064. doi: 10.1371/journal.pntd.0003064

Kelly, D. J., Fuerst, P. A., Ching, W. M., and Richards, A. L. (2009). Scrub typhus: the geographic distribution of phenotypic and genotypic variants of Orientia tsutsugamushi. Clin. Infect. Dis. 48(Suppl. 3), S203-S230. doi: $10.1086 / 596576$

Kim, M., Allen, B., Korhonen, E. A., Nitschké, M., Yang, H. W., Baluk, P., et al. (2016). Opposing actions of angiopoietin-2 on Tie2 signaling and FOXO1 activation. J. Clin. Invest. 126, 3511-3525. doi: 10.1172/JCI84871

Kim, M. K., Kee, S. H., Cho, K. A., Chung, M. H., Lim, B. U., Chang, W. H., et al. (1999). Apoptosis of endothelial cell line ECV304 persistently infected with Orientia tsutsugamushi. Microbiol. Immunol. 43, 751-757. doi: 10.1111/ j.1348-0421.1999.tb02466.x

Koo, J. E., Hong, H. J., Dearth, A., Kobayashi, K. S., and Koh, Y. S. (2012). Intracellular invasion of Orientia tsutsugamushi activates inflammasome in asc-dependent manner. PLoS One 7:e39042. doi: 10.1371/journal.pone.0039042

Kundavaram, A. P., Jonathan, A. J., Nathaniel, S. D., and Varghese, G. M. (2013). Eschar in scrub typhus: a valuable clue to the diagnosis. J. Postgrad. Med. 59, 177-178. doi: 10.4103/0022-3859.118033

Lai, D., Tang, J., Chen, L., Fan, E. K., Scott, M. J., Li, Y., et al. (2018). Group 2 innate lymphoid cells protect lung endothelial cells from pyroptosis in sepsis. Cell Death Dis. 9:369. doi: 10.1038/s41419-018-0412-5

Lefrançais, E., Mallavia, B., Zhuo, H., Calfee, C. S., and Looney, M. R. (2018). Maladaptive role of neutrophil extracellular traps in pathogen-induced lung injury. JCI Insight 3, 1-15. doi: 10.1172/jci.insight.98178

Lefrancais, E., Roga, S., Gautier, V., Gonzalez-de-Peredo, A., Monsarrat, B., Girard, J. P., et al. (2012). IL-33 is processed into mature bioactive forms by neutrophil elastase and cathepsin G. Proc. Natl. Acad. Sci. USA 109, 1673-1678. doi: 10.1073/pnas.1115884109

Li, G. G., Cao, Y. H., Run, Y., Xu, R. X., and Zheng, Z. D. (2016). Inhibition of CD8(+) T cells and elimination of myeloid cells by CD4(+) Foxp3(-) T regulatory type 1 cells in acute respiratory distress syndrome. Clin. Exp. Pharmacol. Physiol. 43, 1191-1198. doi: 10.1111/1440-1681.12656

Lin, S. H., Fu, J., Wang, C. J., Gao, F., Feng, X. Y., Liu, Q., et al. (2016). Inflammation elevated IL-33 originating from the lung mediates inflammation in acute lung injury. Clin. Immunol. 173, 32-43. doi: 10.1016/j.clim.2016.10.014

Longo, D. L., and Harrison, T. R. (2012). Harrison's principles of internal medicine. New York: McGraw-Hill.

Lu, H. L., Huang, X. Y., Luo, Y. F., Tan, W. P., Chen, P. F., and Guo, Y. B. (2018). Activation of M1 macrophages plays a critical role in the initiation of acute lung injury. Biosci. Rep. 38, 1-13. doi: 10.1042/BSR20171555

Lukowski, S. W., Patel, J., Andersen, S. B., Sim, S. L., Wong, H. Y., Tay, J., et al. (2019). Single-cell transcriptional profiling of aortic endothelium identifies a hierarchy from endovascular progenitors to differentiated cells. Cell Rep. 27, 2748-2758.e2743. doi: 10.1016/j.celrep.2019.04.102

Mai, J., Virtue, A., Shen, J., Wang, H., and Yang, X. F. (2013). An evolving new paradigm: endothelial cells--conditional innate immune cells. J. Hematol. Oncol. 6:61. doi: 10.1186/1756-8722-6-61

Matthay, M. A., and Zemans, R. L. (2011). The acute respiratory distress syndrome: pathogenesis and treatment. Annu. Rev. Pathol. 6, 147-163. doi: 10.1146/annurev-pathol-011110-130158

McNeill, E., Crabtree, M. J., Sahgal, N., Patel, J., Chuaiphichai, S., Iqbal, A. J., et al. (2015). Regulation of iNOS function and cellular redox state by macrophage Gch1 reveals specific requirements for tetrahydrobiopterin in NRF2 activation. Free Radic. Biol. Med. 79, 206-216. doi: 10.1016/j.freeradbiomed.2014.10.575

Mendell, N. L., Bouyer, D. H., and Walker, D. H. (2017). Murine models of scrub typhus associated with host control of Orientia tsutsugamushi infection. PLoS Negl. Trop. Dis. 11:e0005453. doi: 10.1371/journal.pntd.0005453

Moron, C. G., Popov, V. L., Feng, H. M., Wear, D., and Walker, D. H. (2001). Identification of the target cells of Orientia tsutsugamushi in human cases of scrub typhus. Mod. Pathol. 14, 752-759. doi: 10.1038/modpathol.3880385
Moussa, M. D., Santonocito, C., Fagnoul, D., Donadello, K., Pradier, O., Gaussem, P., et al. (2015). Evaluation of endothelial damage in sepsis-related ARDS using circulating endothelial cells. Intensive Care Med. 41, 231-238. doi: 10.1007/s00134-014-3589-9

Narasaraju, T., Yang, E., Samy, R. P., Ng, H. H., Poh, W. P., Liew, A. A., et al. (2011). Excessive neutrophils and neutrophil extracellular traps contribute to acute lung injury of influenza pneumonitis. Am. J. Pathol. 179, 199-210. doi: 10.1016/j.ajpath.2011.03.013

Ogawa, M., Satoh, M., Kataoka, M., Ando, S., and Saijo, M. (2017). Nitric oxide enhanced the growth of an obligated intracellular bacterium Orientia tsutsugamushi in murine macrophages. Microb. Pathog. 107, 335-340. doi: 10.1016/j.micpath.2017.04.012

Orfanos, S. E., Mavrommati, I., Korovesi, I., and Roussos, C. (2004). Pulmonary endothelium in acute lung injury: from basic science to the critically ill. Intensive Care Med. 30, 1702-1714. doi: 10.1007/s00134-004-2370-x

Otterdal, K., Janardhanan, J., Astrup, E., Ueland, T., Prakash, J. A., Lekva, T., et al. (2014). Increased endothelial and macrophage markers are associated with disease severity and mortality in scrub typhus. J. Infect. 69, 462-469. doi: 10.1016/j.jinf.2014.06.018

Parikh, S. M. (2017). The angiopoietin-Tie2 signaling axis in systemic inflammation. J. Am. Soc. Nephrol. 28, 1973-1982. doi: 10.1681/ASN.2017010069

Paris, D. H., Phetsouvanh, R., Tanganuchitcharnchai, A., Jones, M., Jenjaroen, K., Vongsouvath, M., et al. (2012). Orientia tsutsugamushi in human scrub typhus eschars shows tropism for dendritic cells and monocytes rather than endothelium. PLoS Negl. Trop. Dis. 6:e1466. doi: 10.1371/journal.pntd.0001466

Paris, D. H., Stephan, F., Bulder, I., Wouters, D., van der Poll, T., Newton, P. N., et al. (2015). Increased nucleosomes and neutrophil activation link to disease progression in patients with scrub typhus but not murine typhus in Laos. PLoS Negl. Trop. Dis. 9:e0003990. doi: 10.1371/journal.pntd.0003990

Park, E. C., Lee, S. Y., Yun, S. H., Choi, C. W., Lee, H., Song, H. S., et al. (2018). Clinical proteomic analysis of scrub typhus infection. Clin. Proteomics 15:6. doi: 10.1186/s12014-018-9181-5

Peter, J. V., Sudarsan, T. I., Prakash, J. A., and Varghese, G. M. (2015). Severe scrub typhus infection: clinical features, diagnostic challenges and management. World J. Crit. Care Med. 4, 244-250. doi: 10.5492/wjccm.v4.i3.244

Rikihisa, Y., and Ito, S. (1982). Entry of Rickettsia tsutsugamushi into polymorphonuclear leukocytes. Infect. Immun. 38, 343-350.

Risso, K., Kumar, G., Ticchioni, M., Sanfiorenzo, C., Dellamonica, J., Guillouet-de Salvador, F., et al. (2015). Early infectious acute respiratory distress syndrome is characterized by activation and proliferation of alveolar T-cells. Eur. J. Clin. Microbiol. Infect. Dis. 34, 1111-1118. doi: 10.1007/ s10096-015-2333-x

Saffarzadeh, M., Juenemann, C., Queisser, M. A., Lochnit, G., Barreto, G., Galuska, S. P., et al. (2012). Neutrophil extracellular traps directly induce epithelial and endothelial cell death: a predominant role of histones. PLoS One 7:e32366. doi: 10.1371/journal.pone.0032366

Sahoo, J. N., Gurjar, M., and Harde, Y. (2016). Acute respiratory failure in scrub typhus patients. Indian J. Crit. Care Med. 20, 480-484. doi: $10.4103 / 0972-5229.188206$

Salje, J. (2017). Orientia tsutsugamushi: a neglected but fascinating obligate intracellular bacterial pathogen. PLoS Pathog. 13:e1006657. doi: 10.1371/ journal.ppat.1006657

Shelite, T. R., Liang, Y., Wang, H., Mendell, N. L., Trent, B. J., Sun, J., et al. (2016). IL-33-dependent endothelial activation contributes to apoptosis and renal injury in Orientia tsutsugamushi-infected mice. PLoS Negl. Trop. Dis. 10:e0004467. doi: 10.1371/journal.pntd.0004467

Shelite, T. R., Saito, T. B., Mendell, N. L., Gong, B., Xu, G., Soong, L., et al. (2014). Hematogenously disseminated Orientia tsutsugamushi-infected murine model of scrub typhus [corrected]. PLoS Negl. Trop. Dis. 8:e2966. doi: 10.1371/ journal.pntd.0002966

Siegel, M. (2019). Acute respiratory distress syndrome: Clinical features, diagnosis, and complications in adults. Waltham, MA: UpToDate.

Silliman, C. C., Curtis, B. R., Kopko, P. M., Khan, S. Y., Kelher, M. R., Schuller, R. M., et al. (2007). Donor antibodies to HNA-3a implicated in TRALI reactions prime neutrophils and cause PMN-mediated damage to human pulmonary microvascular endothelial cells in a two-event in vitro model. Blood 109, 1752-1755. doi: 10.1182/blood-2006-05-025106

Singer, B. D., Mock, J. R., D’Alessio, F. R., Aggarwal, N. R., Mandke, P., Johnston, L., et al. (2016). Flow-cytometric method for simultaneous analysis of mouse 
lung epithelial, endothelial, and hematopoietic lineage cells. Am. J. Physiol. Lung Cell. Mol. Physiol. 310, L796-L801. doi: 10.1152/ajplung.00334.2015

Sônego, F., Castanheira, F. V., Ferreira, R. G., Kanashiro, A., Leite, C. A., Nascimento, D. C., et al. (2016). Paradoxical roles of the neutrophil in sepsis: protective and deleterious. Front. Immunol. 7:155. doi: 10.3389/ fimmu.2016.00155

Song, S. W., Kim, K. T., Ku, Y. M., Park, S. H., Kim, Y. S., Lee, D. G., et al. (2004). Clinical role of interstitial pneumonia in patients with scrub typhus: a possible marker of disease severity. J. Korean Med. Sci. 19, 668-673. doi: 10.3346/jkms.2004.19.5.668

Soong, L., Mendell, N. L., Olano, J. P., Rockx-Brouwer, D., Xu, G., Goez-Rivillas, Y., et al. (2016). An intradermal inoculation mouse model for immunological investigations of acute scrub typhus and persistent infection. PLoS Negl. Trop. Dis. 10:e0004884. doi: 10.1371/journal.pntd.0004884

Soong, L., Shelite, T. R., Xing, Y., Kodakandla, H., Liang, Y., Trent, B. J., et al. (2017). Type 1-skewed neuroinflammation and vascular damage associated with Orientia tsutsugamushi infection in mice. PLoS Negl. Trop. Dis. 11:e0005765. doi: 10.1371/journal.pntd.0005765

Soong, L., Wang, H., Shelite, T. R., Liang, Y., Mendell, N. L., Sun, J., et al. (2014). Strong type 1, but impaired type 2, immune responses contribute to Orientia tsutsugamushi-induced pathology in mice. PLoS Negl. Trop. Dis. 8:e3191. doi: 10.1371/journal.pntd.0003191

Stiehl, T., Thamm, K., Kaufmann, J., Schaeper, U., Kirsch, T., Haller, H., et al. (2014). Lung-targeted RNA interference against angiopoietin-2 ameliorates multiple organ dysfunction and death in sepsis. Crit. Care Med. 42, e654-e662. doi: 10.1097/CCM.0000000000000524

Summers, C., Rankin, S. M., Condliffe, A. M., Singh, N., Peters, A. M., and Chilvers, E. R. (2010). Neutrophil kinetics in health and disease. Trends Immunol. 31, 318-324. doi: 10.1016/j.it.2010.05.006

Sunyakumthorn, P., Paris, D. H., Chan, T. C., Jones, M., Luce-Fedrow, A., Chattopadhyay, S., et al. (2013). An intradermal inoculation model of scrub typhus in Swiss CD-1 mice demonstrates more rapid dissemination of virulent strains of Orientia tsutsugamushi. PLoS One 8:e54570. doi: 10.1371/journal. pone. 0054570

Sunyakumthorn, P., Somponpun, S. J., Im-Erbsin, R., Anantatat, T., Jenjaroen, K., Dunachie, S. J., et al. (2018). Characterization of the rhesus macaque (Macaca mulatta) scrub typhus model: susceptibility to intradermal challenge with the human pathogen Orientia tsutsugamushi Karp. PLoS Negl. Trop. Dis. 12:e006305. doi: 10.1371/journal.pntd.0006305

Suwanpradid, J., Shih, M., Pontius, L., Yang, B., Birukova, A., Guttman-Yassky, E., et al. (2017). Arginasel deficiency in monocytes/macrophages upregulates inducible nitric oxide synthase to promote cutaneous contact hypersensitivity. J. Immunol. 199, 1827-1834. doi: 10.4049/jimmunol.1700739

Tantibhedhyangkul, W., Prachason, T., Waywa, D., El Filali, A., Ghigo, E., Thongnoppakhun, W., et al. (2011). Orientia tsutsugamushi stimulates an original gene expression program in monocytes: relationship with gene expression in patients with scrub typhus. PLoS Negl. Trop. Dis. 5:e1028. doi: 10.1371/journal.pntd.0001028
Tsay, R. W., and Chang, F. Y. (2002). Acute respiratory distress syndrome in scrub typhus. QJM 95, 126-128. doi: 10.1093/qjmed/95.2.126

Valbuena, G., and Walker, D. H. (2012). Approaches to vaccines against Orientia tsutsugamushi. Front. Cell. Infect. Microbiol. 2:170. doi: 10.3389/fcimb.2012.00170

van Meurs, M., Kümpers, P., Ligtenberg, J. J., Meertens, J. H., Molema, G., and Zijlstra, J. G. (2009). Bench-to-bedside review: angiopoietin signalling in critical illness - a future target? Crit. Care 13:207. doi: 10.1186/cc7153

Varghese, G. M., Janardhanan, J., Trowbridge, P., Peter, J. V., Prakash, J. A., Sathyendra, S., et al. (2013). Scrub typhus in South India: clinical and laboratory manifestations, genetic variability, and outcome. Int. J. Infect. Dis. 17, e981-e987. doi: 10.1016/j.ijid.2013.05.017

Varghese, G. M., Trowbridge, P., Janardhanan, J., Thomas, K., Peter, J. V., Mathews, P., et al. (2014). Clinical profile and improving mortality trend of scrub typhus in South India. Int. J. Infect. Dis. 23, 39-43. doi: 10.1016/j. ijid.2014.02.009

Vergadi, E., Vaporidi, K., Theodorakis, E. E., Doxaki, C., Lagoudaki, E., Ieronymaki, E., et al. (2014). Akt2 deficiency protects from acute lung injury via alternative macrophage activation and miR-146a induction in mice. J. Immunol. 192, 394-406. doi: 10.4049/jimmunol.1300959

Wang, C. C., Liu, S. F., Liu, J. W., Chung, Y. H., Su, M. C., and Lin, M. C. (2007). Acute respiratory distress syndrome in scrub typhus. Am. J .Trop. Med. Hyg. 76, 1148-1152. doi: 10.4269/ajtmh.2007.76.1148

Weitzel, T., Dittrich, S., López, J., Phuklia, W., Martinez-Valdebenito, C., Velásquez, K., et al. (2016). Endemic scrub typhus in South America. N. Engl. J. Med. 375, 954-961. doi: 10.1056/NEJMoa1603657

Witter, A. R., Okunnu, B. M., and Berg, R. E. (2016). The essential role of neutrophils during infection with the intracellular bacterial pathogen Listeria monocytogenes. J. Immunol. 197, 1557-1565. doi: 10.4049/jimmunol.1600599

Xu, G., Mendell, N. L., Liang, Y., Shelite, T. R., Goez-Rivillas, Y., Soong, L., et al. (2017). Correction: CD8+ T cells provide immune protection against murine disseminated endotheliotropic Orientia tsutsugamushi infection. PLoS Negl. Trop. Dis. 11:e0006127. doi: 10.1371/journal.pntd.0006127

Yun, J. H., Koo, J. E., and Koh, Y. S. (2009). Mitogen-activated protein kinases are involved in tumor necrosis factor alpha production in macrophages infected with Orientia tsutsugamushi. Microbiol. Immunol. 53, 349-355. doi: 10.1111/j.1348-0421.2009.00127.x

Conflict of Interest Statement: The authors declare that the research was conducted in the absence of any commercial or financial relationships that could be construed as a potential conflict of interest.

Copyright (C) 2019 Trent, Fisher and Soong. This is an open-access article distributed under the terms of the Creative Commons Attribution License (CC BY). The use, distribution or reproduction in other forums is permitted, provided the original author(s) and the copyright owner(s) are credited and that the original publication in this journal is cited, in accordance with accepted academic practice. No use, distribution or reproduction is permitted which does not comply with these terms. 